\title{
Influência dos parâmetros de fabricação nas Propriedades Físicas e Mecânicas de Paineis de Partícula de Média Densidade
}

\author{
Influence of Fabrication Parameters on the \\ Physical and Mechanical Properties of \\ Medium Density Particleboards
}

\author{
Diogo Aparecido Lopes Silva ${ }^{1}$, Vinicius Borges de Moura Aquino ${ }^{2}$, \\ Francisco Antonio Rocco Lahr ${ }^{3}$, André Luis Christoforo ${ }^{4}$
}

\footnotetext{
${ }^{1}$ Departamento de Engenharia de Produção de Sorocaba, CCGT/UFSCar, Rodovia João Leme dos Santos (SP-264), Km 110, CEP: 18052-780 , Sorocaba, SP, Brasil.

${ }^{2}$ Faculdade de Tecnologia, IEA/UNIFESSPA, Rua Geraldo Ramalho, 33, CEP 68560-000, Santana do Araguaia, PA, Brasil.

${ }^{3}$ Departamento de Engenharia de Estruturas, EESC/USP, Av. Trabalhador São-carlense, 400, Parque Arnold Schimit, CEP 13566-590, São Carlos, SP, Brasil.

${ }^{4}$ Departamento de Engenharia Civil, CCET/UFSCar, Rodovia Washington Luís (SP-310), km 235, CEP 36307-352, São

Carlos, SP, Brasil.

e-mail: diogo.apls@ufscar.br, aquino.vini@hotmail.com, frocco@sc.usp.br, alchristoforo@gmail.com
}

\section{RESUMO}

A presente pesquisa tem por objetivo avaliar a influência de três fatores de produção (densidade nominal, temperatura e pressão) na produção de paineis de partículas de média densidade da madeira de Eucalyptus saligna com resina ureia-formaldeído e catalisadores e verificar se os paineis atendem ao requisito normativo da ABNT NBR 14810. A influência dos fatores nos paineis MDP foram analisados por intermédio de 12 tratamentos experimentais. Foram fabricadas 48 chapas ao total, com 4 chapas com dimensões $300 \mathrm{~mm} \times 300$ mm x $15 \mathrm{~mm}$, determinando 7 propriedades físicas e mecânicas, totalizando 336 determinações experimentais. Foram avaliadas as seguintes propriedades: teor de umidade, densidade aparente, inchamento em espessura após $2 \mathrm{~h}$ e $24 \mathrm{~h}$, tração perpendicular, módulo de elasticidade e módulo de resistência. Os tratamentos, em grande parte, atenderam aos requisitos normativos, com três tratamentos atendendo plenamente. Para a maioria das propriedades os fatores de produção não foram significativos do ponto de vista estatístico, possibilitando que possa haver redução de algum fator a fim de manter o mesmo desempenho atendendo aos requisitos normativos.

Palavras-chave: Painéis de partículas de média densidade (MDP), Eucalyptus, fator de produção, desempenho, análise de variância (ANOVA).

\section{ABSTRACT}

This research aims to evaluate the influence of three production factors (nominal density, temperature and pressure) on the production of medium density particleboard of Eucalyptus saligna wood with urea formaldehyde resin and catalysts and to verify if the panels meet the normative requirement of ABNT NBR 14810. The influence of factors on MDP panels were analyzed through 12 experimental treatments. A total of 48 sheets were manufactured, with 4 sheets with dimensions $300 \mathrm{~mm}$ x $300 \mathrm{~mm}$ x $15 \mathrm{~mm}$, determining 7 physical and mechanical properties, totaling 336 experimental determinations. The following properties were evaluated: moisture content, apparent density, thickness swelling after $2 \mathrm{~h}$ and $24 \mathrm{~h}$, perpendicular tensile, elastic modulus and resistance modulus. The treatments largely met normative requirements, with three treatments fully meeting. For most properties the factors of production were not statistically significant, allowing some factor to be reduced in order to maintain the same performance meeting the normative requirements.

Keywords: Medium density particleboard, Eucalyptus, production factor, performance, analysis of variance 


\section{INTRODUÇÃO}

A madeira, um material natural, é utilizada nas atividades humanas desde tempos remotos na construção de abrigos e ferramentas para agricultura e pecuária [1,2]. Uma alternativa ao uso de madeira serrada em edificações com fins estruturais, móveis e outras aplicações consiste no uso de produtos engenheirados à base de madeira, como painéis compensados, OSB, MDF e MDP [3-7].

Estes painéis são constituídos de madeira processada (partículas, lascas ou lamelas) e resina, unidas à quente sob pressão [8-10]. As resinas utilizadas no processo de fabricação podem ser acetato de polivinila (PVA), fenol formaldeído (FF), resorcinol formaldeído (RF), ureia formaldeído (UF), difenilmetano diisocianato (MDI) e poliuretana (PU) à base de óleos de origem vegetal [3,11-16], destacando-se a resina ureia formaldeído, utilizada na fabricação de paineis comerciais [12,17-19].

Dentre os paineis engenheirados à base de madeira, destaca-se o uso de painéis MDP (Medium Density Particleboard), utilizados na indústria para confecção de móveis [20-23] e, quando associados com outros produtos engenheirados, como lamelas e lascas de madeira, podem ser utilizados para fins estruturais [17,24].

As madeiras utilizadas para confecção de painéis para uso comercial são as espécies do gênero Pinus spp.e Eucalyptus spp., as quais são espécies de produção em larga escala e ditas "madeiras de reflorestamento" [25,26]. Dentre estas, destaca-se a espécie Eucalyptus saligna, espécie de reflorestamento, com possibilidade de uso em fins estruturais, classificada na classe C30 das espécies folhosas, possibilitando seu uso em painéis de madeira [27-31].

O processo de fabricação dos painéis de madeira consiste na união de madeira processada e resina, devidamente homogeneizada, onde deve haver uma prensagem à quente a fim de que haja a consolidação do material para conferir resistência e durabilidade [20,24]. Considerando que a temperatura de prensagem, a pressão aplicada durante a prensagem e a densidade do material particulado de madeira são fatores que influenciam nas propriedades físicas e mecânicas, a combinação ótima de tais fatores pode levar a painéis com propriedades satisfatórias, com valores superiores aos requisitos mínimos dispostos em normas, como a NBR 14810 [32], CS 236-66 [33] e EN-312 [34]. Também deve-se destacar que tais fatores, em escala industrial, possuem grande relevância na composição dos custos de produção e eficiência do processo produtivo, fazendo assim que possa haver redução de custos no processo produtivo, bem como aumento da eficiência do produto.

Desta forma, destacam-se na literatura os trabalhos de LONGO et al. [35], SILVA et al. [5], IHNÁT et al.[36], SCATOLINO et al. [37] e SOUZA et al.[38], onde utilizam material processado de madeira e resina ureia formaldeído para confecção de painéis, onde estes atingiram os requisitos normativos estabelecidos pela NBR 14810 [32] ou EN-300 [34]. Também destaca-se os trabalhos de SILVA et al. [39] e PAN et al. [16] versando sobre paineis MDP feitos resina ureia-formaldeído e partículas de madeira do gênero Eucalyptus. Porém, não se verifica na literatura pesquisas versando sobre a influência dos fatores temperatura, pressão e densidade do material particulado na confecção de painéis MDP com resina ureia-formaldeído e partículas de madeira de Eucalyptus saligna.

Assim, a presente pesquisa tem por objetivo avaliar a influência dos parâmetros de fabricação nas propriedades físicas e mecânicas de painéis de partícula de média densidade (MDP) utilizando resíduos da madeira de Eucalyptus saligna e resina ureia formaldeído, avaliando diferentes situações de pressão, temperatura e densidade. Também será avaliado se as condições ótimas dos parâmetros de fabricação atendem os requisitos normativos dispostos na NBR 14810 [32].

\section{MATERIAIS E MÉTODOS}

Os fatores e os respectivos níveis investigados na fabricação dos painéis de partículas da presente pesquisa consistiram na pressão de prensagem [P] $(3,0 ; 3,5$ e 4,0 MPa), temperatura de prensagem [T] $(165 \mathrm{e}$ $\left.170{ }^{\circ} \mathrm{C}\right)$ e na densidade nominal $[\rho]\left(0,5\right.$ e $\left.0,7 \mathrm{~g} / \mathrm{cm}^{3}\right)$, resultando em 12 tratamentos [Tr] experimentais distintos, assim como ilustrado na Tabela 1 .

Tabela 1: Descrição dos fatores de produção investigados.

\begin{tabular}{l|l|l|l}
\hline $\begin{array}{l}\text { TRATAMENTO } \\
{[\mathrm{TR}]}\end{array}$ & $\begin{array}{l}\text { PRESSÃO [P] - } \\
\mathbf{M P a}\end{array}$ & $\begin{array}{l}\text { TEMPERATURA } \\
{[\mathrm{T}]-{ }^{\circ} \mathbf{C}}\end{array}$ & $\begin{array}{l}\text { DENSIDADE [D] } \\
-\mathbf{g} / \mathbf{c m}^{3}\end{array}$ \\
\hline 1 & 3,0 & 165 & 0,5 \\
\hline
\end{tabular}




\begin{tabular}{l|l|l|l}
\hline 2 & 3,0 & 165 & 0,7 \\
\hline 3 & 3,0 & 170 & 0,5 \\
\hline 4 & 3,0 & 170 & 0,7 \\
\hline 5 & 3,5 & 165 & 0,5 \\
\hline 6 & 3,5 & 165 & 0,7 \\
\hline 7 & 3,5 & 170 & 0,5 \\
\hline 8 & 3,5 & 170 & 0,7 \\
\hline 9 & 4,0 & 165 & 0,5 \\
\hline 10 & 4,0 & 165 & 0,7 \\
\hline 12 & 4,0 & 170 & 0,5 \\
\hline
\end{tabular}

As propriedades físicas e mecânicas, obtidas de acordo com as premissas e métodos de cálculo da norma brasileira ABNT NBR 14810 (2013), consistiram no teor de umidade [TU], densidade aparente [ $\left.\rho_{\text {ap }}\right]$, inchamento em espessura após 2 horas [I-2h] e após 24 horas [I-24h] de imersão em água, tração perpendicular [TP] e módulos de elasticidade [MOE] e de ruptura [MOR] na flexão estática.

Para cada tratamento foram fabricados 4 painéis, o que resultou em 48 chapas de dimensões $300 \mathrm{~mm}$ $\times 300 \mathrm{~mm} \times 15 \mathrm{~mm}$. Por serem 4 propriedades físicas e 3 mecânicas, ao todo foram obtidas 336 determinações experimentais.

Modelos de regressão (Equação 1) foram utilizados para estabelecer relações entre os três fatores investigados (temperatura $[\mathrm{T}]$, pressão $[\mathrm{P}]$ e densidade nominal $[\mathrm{D}]$ ) e as propriedades físicas e mecânicas de interesse, possibilitando determinar combinações ótimas dos três fatores que resultam em valores máximos ou mínimos das propriedades avaliadas. O coeficiente de determinação $\mathrm{R}^{2}$ foi utilizado para julgar a qualidade dos ajustes.

$$
\mathrm{Y}=\beta 0+\beta 1 \cdot \mathrm{P}+\beta 2 \mathrm{~T}+\beta 3 \cdot \rho+\beta 4 \cdot \mathrm{P} \cdot \mathrm{T}+\beta 5 \cdot \mathrm{P} \cdot \rho+\beta 6 \cdot \mathrm{T} \cdot \rho+\beta 7 \cdot \mathrm{P} \cdot \mathrm{T} \cdot \rho+\varepsilon
$$

Da Equação 1, Y consiste nas propriedades físicas e mecânicas, $\beta$ i são os coeficientes obtidos pelo método dos mínimos quadrados e $\varepsilon$ é o erro aleatório cometido nas estimativas.

A análise de variância (ANOVA), ao nível de $5 \%$ de significância ( $\alpha$ ), foi utilizada com o objetivo de verificar a significância de cada termo componente da Equação 1, possibilitando julgar a influência dos fatores isolados assim como das suas interações (produtos entre fatores distintos) em cada propriedade medida. Pela formulação das hipóteses da ANOVA, P-valor (probabilidade P) igual ou superior ao nível de significância adotado $(0,05)$ implica que termos ou modelo são considerados não significativos, e significativos em caso contrário $(\mathrm{P}<0,05)$.

Gráficos de interação entre os fatores foram utilizados para interpretar os efeitos das interações acusadas significativas pela ANOVA na estimativa de uma determinada propriedade (física ou mecânica).

Os paineis MDP desta pesquisa são compostos de partículas de madeira da espécie Eucalyptus saligna processadas em um gerador de partículas localizado no Laboratório de Madeiras e de Estruturas de Madeira (LaMEM), Escola de Engenharia de São Carlos (EESC), Universidade de São Paulo (USP), com teor de umidade igual a $12 \%$ [40]. As partículas foram classificadas em dois grupos: material de camada interna (CI) e de camada externa (CE). Na CE de cada painel produzido foram aplicadas partículas com dimensões de até $1,18 \mathrm{~mm}$, enquanto que na CI foram utilizadas partículas com dimensões de 1,18 a 2,38 $\mathrm{mm}$. Os paineis foram fabricados com espessura igual a $15 \mathrm{~mm}$, com densidades nominais descritas na Tabela 1. As camadas externas tiveram espessura de $2,5 \mathrm{~mm}$ cada e a camada interna possuía $10 \mathrm{~mm}$ de espessura.

A resina utilizada foi a ureia-formaldeído, com teor igual a $12 \%$ em relação ao peso seco das partículas. A resina utilizada apresentou razão molar formaldeído/ureia de 1,35, teor de sólidos de $64,0 \%$, e densidade de $1,27 \mathrm{~g} / \mathrm{cm}^{3}$. Além da resina ureia-formaldeído, foram adicionados os seguintes catalisadores: emulsão de parafina e sulfato de amônio, cada um com teor de $1 \%$ em relação à massa seca das partículas.

A resina e os catalizadores foram homogeneizados mecanicamente com as partículas em duas etapas, com a primeira etapa sendo a mistura com o material da camada externa e a segunda etapa sendo a mistura com o material da camada interna. Após a homogeneização, o material foi levado a uma forma de madeira para pré-prensagem $(1 \mathrm{MPa})$.

Após a pré-compactação, o colchão de partículas foi levado à prensa hidráulica a quente, com a tem- 
peratura e pressão variando de acordo com o tratamento disposto na Tabela 1.

Após a prensagem à quente, os paineis foram levados para uma sala com temperatura e umidade constante por 72 horas a fim de estabilização e cura completa do painel. Após a cura, os paineis foram esquadrejados e extraídos os corpos de prova, com dimensões de $250 \mathrm{~mm}$ x $50 \mathrm{~mm}$ para avaliação do MOR e MOE e de $50 \mathrm{~mm} \times 50 \mathrm{~mm}$ para as demais propriedades [32].

Também foi avaliado as emissões ao ar de formaldeído livre. Para isto, foi feito o monitoramento das emissões ao ar de formaldeído livre (em g), foi realizada uma estimativa em função do peso (em g) e do teor de umidade (em \%) de cada painel produzido, e considerando a classe E2 de painéis pela norma NBR 148102:2013 [32]. Painéis aglomerados classificados como E2 devem apresentar teor de emissão de formaldeído livre superior a $8 \mathrm{mg}$ e inferior ou igual a $20 \mathrm{mg}$ para cada $100 \mathrm{~g}$ de painel seco [32].

\section{RESULTADOS E DISCUSSÕES}

A Tabela 2 apresenta os valores médios $(\bar{x})$ e os respectivos coeficientes de variação [CV] das propriedades físicas dos painéis fabricados segundo os 12 tratamentos delineados nessa pesquisa, e na Tabela 3 são apresentados os resultados das propriedades mecânicas.

Tabela 2: Resultados das propriedades físicas dos painéis fabricados.

\begin{tabular}{|c|c|c|c|c|c|c|c|c|}
\hline \multirow[t]{2}{*}{ TR } & \multirow{2}{*}{$\begin{array}{l}\text { TU (\%) } \\
\bar{x}\end{array}$} & \multicolumn{3}{|c|}{$P(g / c m 3)$} & \multicolumn{2}{|l|}{ I-2H (\%) } & \multicolumn{2}{|c|}{$\mathrm{I}-24 \mathrm{H}(\%)$} \\
\hline & & $\mathrm{CV}(\%)$ & $\bar{x}$ & $\mathrm{CV}(\%)$ & $\bar{x}$ & CV(\%) & $\bar{x}$ & $\mathrm{CV}(\%)$ \\
\hline 1 & 36,04 & 16,01 & 0,56 & 1,84 & 19,44 & 50,35 & 31,12 & 60,83 \\
\hline 2 & 5,84 & 7,82 & 0,59 & 3,43 & 2,64 & 38,42 & 7,79 & 30,31 \\
\hline 3 & 31,07 & 48,19 & 0,38 & 8,11 & 8,02 & 42,19 & 28,95 & 55,14 \\
\hline$\underline{4}$ & 5,13 & 7,42 & 0,63 & 7,77 & 2,59 & 41,38 & 6,66 & 30,53 \\
\hline 5 & 33,13 & 23,26 & 0,59 & 3,43 & 25,82 & 36,02 & 39,44 & 57,53 \\
\hline 6 & 5,84 & 7,82 & 0,65 & 15,98 & 1,84 & 37,89 & 5,51 & 26,51 \\
\hline 7 & 25,68 & 28,97 & 0,59 & 5,12 & 12,80 & 74,20 & 26,50 & 29,46 \\
\hline 8 & 5,14 & 7,84 & 0,59 & 5,12 & 1,29 & 43,51 & 5,30 & 24,95 \\
\hline 9 & 28,39 & 20,13 & 0,37 & 3,88 & 4,77 & 50,21 & 17,39 & 75,26 \\
\hline 10 & 7,22 & 3,25 & 0,69 & 18,60 & 1,32 & 48,86 & 4,37 & 12,05 \\
\hline 11 & 27,87 & 13,24 & 0,62 & 3,44 & 7,24 & 39,22 & 21,31 & 20,27 \\
\hline 12 & 5,38 & 46,77 & 0,58 & 6,63 & 1,38 & 53,96 & 5,97 & 48,10 \\
\hline
\end{tabular}

Tabela 3: Resultados das propriedades físicas dos painéis.

\begin{tabular}{l|llll|l|l}
\hline TR & TP (MPa) & \multicolumn{3}{c}{ MOE (MPa) } & \multicolumn{3}{c}{ MOR (MPa) } \\
& $\bar{x}$ & $\mathbf{C V}(\%)$ & $\bar{x}$ & \multicolumn{1}{c}{ CV(\%) } & \multicolumn{1}{c}{$\bar{c}$ CV(\%) } \\
\hline 1 & 0,18 & 19,07 & 1524 & 27,71 & 8,39 & 24,91 \\
\hline 2 & 0,51 & 22,36 & 1230 & 21,58 & 11,72 & 9,22 \\
\hline 3 & 0,21 & 3,10 & 654 & 24,46 & 3,12 & 44,64 \\
\hline 4 & 0,46 & 36,65 & 1128 & 14,04 & 10,47 & 13,63 \\
\hline 5 & 0,28 & 12,93 & 1684 & 33,85 & 12,33 & 25,11 \\
\hline 6 & 0,63 & 23,71 & 1230 & 21,58 & 11,72 & 9,22 \\
\hline 7 & 0,19 & 21,03 & 1566 & 14,85 & 7,48 & 27,54 \\
\hline 8 & 0,44 & 7,08 & 1566 & 14,85 & 9,70 & 14,77 \\
\hline 9 & 0,28 & 4,49 & 860 & 25,27 & 8,50 & 52,76 \\
\hline 10 & 0,38 & 39,25 & 2295 & 26,80 & 12,83 & 6,28 \\
\hline 11 & 0,30 & 4,25 & 1730 & 15,54 & 11,15 & 19,66 \\
\hline 12 & 0,57 & 16,85 & 1730 & 15,54 & 14,43 & 5,91 \\
\hline
\end{tabular}


Observando os paineis produzidos quanto ao requisito normativo de densidade, todos os tratamentos, exceto os tratamentos 3 e 9, atenderam ao requisito pela Norma Brasileira ABNT NBR 14810 [32], que define que a densidade de um painel MDP deve estar entre $0,4 \mathrm{~g} / \mathrm{cm}^{3}$ e $0,8 \mathrm{~g} / \mathrm{cm}^{3}$.

Quanto ao requisito de inchamento em espessura, o requisito normativo preconiza que, paineis com espessura de $15 \mathrm{~mm}$, classificados como não estruturais e para uso interno em condições secas (Tipo P2), classificação esta dos paineis confeccionados nesta pesquisa, deve ser inferior a $18 \%$. Desta forma, os tratamentos 2, 4, 6, 8, 10 e 12 atenderam este requisito com baixos valores no coeficiente de variação, com valores inferiores a $50 \%$.

Sobre a propriedade do teor de umidade, definido como o percentual de água desprendida do material quando este é submetido a temperatura superior a $100,0^{\circ} \mathrm{C}$, a Norma dispõe que o teor de umidade deve variar de 5,0 a 13,0\% [32]. Assim, apenas os tratamentos 2, 4, 6, 8, 10 e 12 atenderam este requisito.

Considerando os requisitos resistência à flexão estática [MOR] e módulo de elasticidade [MOE], o requisito normativo para paineis de $15 \mathrm{~mm}$ de espessura, classificados como Tipo P2, a resistência mínima na flexão estática deve ser de 11,0 MPa, enquanto que o módulo de elasticidade mínimo deve ser de $1600 \mathrm{MPa}$ [32]. Observando os resultados, verifica-se que os tratamentos 2, 5, 6, 10,11 e 12 atenderam ao requisito para MOR e os tratamentos 5, 10, 11 e 12 atenderam ao requisito normativo para MOE.

Quanto à resistência na tração perpendicular, cuja propriedade mede a resistência que um corpo-deprova oferece quando é submetido a uma força de tração perpendicularmente à sua superfície, no sentido do comprimento do corpo-de-prova até a sua ruptura. O requisito normativo para chapas de $15 \mathrm{~mm}$ e classificadas como Tipo P2, a resistência mínima na tração deve ser de 0,35 MPa [32]. Os tratamentos 2, 4, 6, 8, 10 e 12 atenderam ao requisito normativo.

Comparando com dados dispostos na literatura, a pesquisa realizada por SILVA et al. [39] avaliou paineis MDP com a espécie de madeira Eucalyptus grandis executados com densidade nominal de $0,7 \mathrm{~g} / \mathrm{cm}^{3}$, temperatura de $160^{\circ}$ e pressão igual a 3,94 MPa, condições próximas ao tratamento 10 da presente pesquisa. As propriedades I-2h, I-24h, TP, MOR e MOE desta pesquisa, considerando o desempenho, foram superiores ao estudo de SILVA et al.[39], destacando-se os valores de inchamento I-2h e I-24h desta pesquisa 14 e 5 vezes, respectivamente, menor em relação à pesquisa de SILVA et al.[39].

As Equações de 2 a 8 expressam os modelos de regressão que relacionam as propriedades físicas e mecânicas com os três fatores idealizados $(\mathrm{P}, \mathrm{T}$ e $\rho)$, encontrando-se sublinhados os termos $(\mathrm{P}$-valor $<0,05)$ considerados significativos pela ANOVA (5\% de significância).

$$
\begin{aligned}
& \mathrm{TU}=2740-638 \cdot \mathrm{P}-15.4 \cdot \mathrm{T}-4048 \cdot \rho+3.68 \cdot \mathrm{P} \cdot \mathrm{T}+967 \cdot \mathrm{P} \cdot \rho+22.8 \cdot \mathrm{T} \cdot \rho-5.59 \cdot \mathrm{P} \cdot \mathrm{T} \cdot \rho\left[\mathrm{R}^{2}=85,09 \%\right] \\
& \rho \mathrm{p}=217.1-63.81 \cdot \mathrm{P}-1.295 \cdot \mathrm{T}-331.9 \cdot \rho+0.3811 \cdot \mathrm{P} \cdot \mathrm{T}+98.2 \cdot \mathrm{P} \cdot \rho+1.984 \cdot \mathrm{T} \cdot \rho-0.5864 \cdot \mathrm{P} \cdot \mathrm{T} \cdot \rho \quad[\mathrm{R} 2= \\
& 68,57 \%] \\
& \mathrm{I}-2 \mathrm{~h}=6632-1642 \cdot \mathrm{P}-38.9 \cdot \mathrm{T}-9438 \cdot \rho+9.66 \cdot \mathrm{P} \cdot \mathrm{T}+2339 \cdot \mathrm{P} \cdot \rho+55.3 \cdot \mathrm{T} \cdot \rho-13.8 \cdot \mathrm{P} \cdot \mathrm{T} \cdot \rho \quad\left[\mathrm{R}^{2}=43,64 \%\right](4) \\
& \mathrm{I}-24 \mathrm{~h}=2335-517 \cdot \mathrm{P}-12.8 \cdot \mathrm{T}-2863 \cdot \rho+2.9 \cdot \mathrm{P} \cdot \mathrm{T}+604 \cdot \mathrm{P} \cdot \rho+15.5 \cdot \mathrm{T} \cdot \rho-3.4 \cdot \mathrm{P} \cdot \mathrm{T} \cdot \rho \quad[\mathrm{R} 2=39,65 \%] \\
& \mathrm{TP}=-73.0+20.8 \cdot \mathrm{P}+0.426 \cdot \mathrm{T}+144.7 \cdot \rho-0.1218 \cdot \mathrm{P} \cdot \mathrm{T}-40.8 \cdot \mathrm{P} \cdot \rho-0.845 \cdot \mathrm{T} \cdot \rho+0.240 \cdot \mathrm{P} \cdot \mathrm{T} \cdot \rho \quad[\mathrm{R} 2= \\
& 65,45 \%] \\
& \mathrm{MOE}=851042-244236 \cdot \mathrm{P}-5047 \cdot \mathrm{T}-1290003 \cdot \rho+1450 \cdot \mathrm{P} \cdot \mathrm{T}+372245 \cdot \mathrm{P} \cdot \rho+7642 \cdot \mathrm{T} \cdot \mathrm{D}-2204 \cdot \mathrm{P} \cdot \mathrm{T} \cdot \rho \\
& {[\mathrm{R} 2=58,48 \%]} \\
& \mathrm{MOR}=2631-681 \cdot \mathrm{P}-15.87 \cdot \mathrm{T}-3250 \cdot \rho+4.12 \cdot \mathrm{P} \cdot \mathrm{T}+840 \cdot \mathrm{P} \cdot \rho+19.7 \cdot \mathrm{T} \cdot \rho-5.06 \cdot \mathrm{P} \cdot \mathrm{T} \cdot \rho \quad[\mathrm{R} 2= \\
& 66,592 \%]
\end{aligned}
$$

Das Equações de 2 a 8, os coeficientes de determinação variaram de 39,65 a 85,09\%, cabendo destacar que variabilidade dos resultados impacta diretamente nas propriedades estimadas.

Para o teor de umidade (TU), o único fator considerado significativo foi a densidade nominal $(\rho)$, ou seja, os dois demais fatores (P e T) e a interação dos fatores não foram considerados significativos na estimativa dos valores do TU, implicando que o uso de quaisquer níveis para a pressão (3,5 ou 4,0 MPa) e para a temperatura $\left(165\right.$ ou $170{ }^{\circ} \mathrm{C}$ ) implicam em valores equivalentes no teor de umidade. Da Equação 2, aumento no valor da densidade nominal de 0,50 para $0,70 \mathrm{~g} / \mathrm{cm}^{3}$ implica em redução no valor do teor de umidade, fato esse justificado pela menor quantidade de vazios presente no painel de maior densidade $[5,24,39,41,42]$. 
Para a densidade aparente (Equação 3), a densidade nominal e a interação entre os fatores pressão e temperatura (P·T) afetaram de forma significativa nos valores dessa propriedade. Aumento na densidade nominal impacta na redução da densidade aparente. Da interação dos fatores $(\mathrm{P} \cdot \mathrm{T})$, para temperatura de $165^{\circ} \mathrm{C}$, a densidade aparente sofre redução de 3 para $4 \mathrm{MPa}$ de pressão, e para a temperatura de $170^{\circ} \mathrm{C}$, a $\rho_{\text {ap }}$ sofre aumento de 3 para $4 \mathrm{MPa}$ (Figura 1a). O menor valor da $\rho_{\text {ap }}\left(0,51 \mathrm{~g} / \mathrm{cm}^{3}\right)$ foi proveniente da combinação de 3 MPa de pressão e $170{ }^{\circ} \mathrm{C}$ de temperatura de prensagem, e o maior valor $\left(0,62 \mathrm{~g} / \mathrm{cm}^{3}\right)$ foi obtido da combinação de $\mathrm{P}=4 \mathrm{MPa}$ e $\mathrm{T}=170{ }^{\circ} \mathrm{C}$.
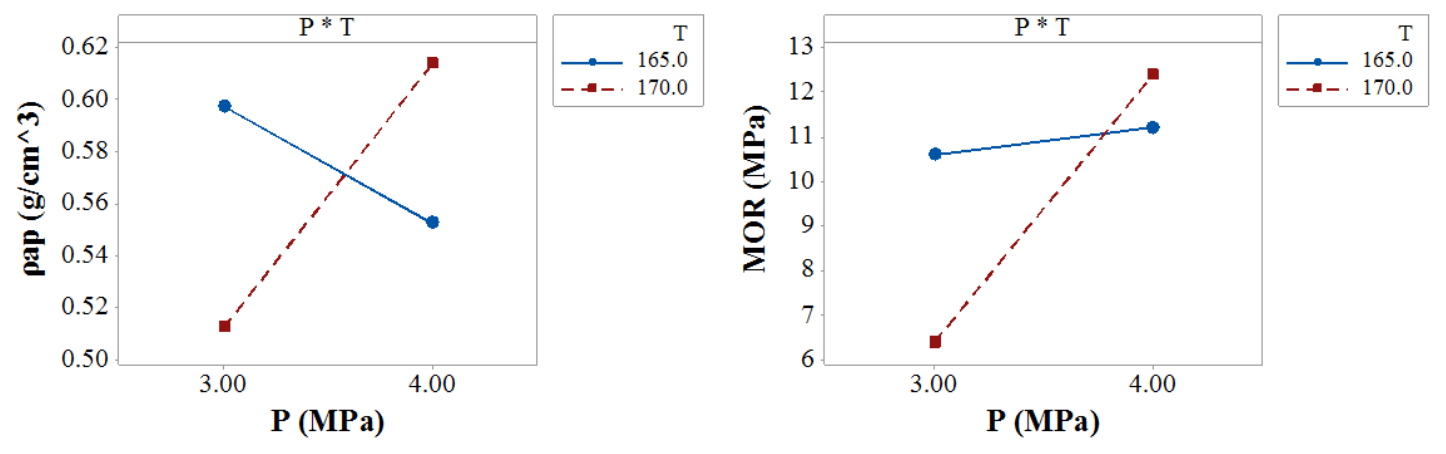

(a)

(b)

Figura 1: Gráficos de interação entre fatores - $\rho$ ap (a), MOR (b).

Para os inchamentos em espessura após 2 (I-2h) e 24 (I-24h) horas de imersão em água e para a tração perpendicular (TP), assim como ocorreu com o teor de umidade, o único fator considerado significativo foi a densidade nominal (Equações 4, 5 e 6). Aumentos na densidade nominal promovem reduções nos valores do inchamento após 2 ou 24 horas e aumentos nos valores da tração perpendicular, assim como esperado.

Para módulo de elasticidade (MOE), nem os fatores e nem as interações entres esses impactaram de forma significativa (Equação 7), implicando que os níveis estabelecidos para a pressão (3,5; 4,0 MPa), temperatura $\left(165 ; 170{ }^{\circ} \mathrm{C}\right)$ e densidade nominal $\left(0,50 ; 0,70 \mathrm{~g} / \mathrm{cm}^{3}\right)$ foram considerados relativamente próximos.

No caso do módulo de resistência na flexão estática (MOR), os três fatores individuais e a interação entre a pressão e a temperatura foram considerados significativos (Equação 8). Aumentos nas três variáveis $(\mathrm{P}, \mathrm{T}$ e $\rho$ ) implicam na redução do MOR, e da interação entre os fatores, para os dois níveis de temperatura $\left(165 ; 170{ }^{\circ} \mathrm{C}\right)$, os valores da resistência na flexão estática sofreram aumentos de 3 para $4 \mathrm{MPa}$ de pressão, sendo notado maior variação para a pressão de $4 \mathrm{MPa}$.

Nos casos onde a temperatura e a pressão de prensagem não foram significativos, é importante destacar que os resultados da presente pesquisa se mostram ainda mais relevantes, pois uma fábrica produz uma imensa quantidade de painéis diariamente, e uma pequena redução na temperatura $\left(170^{\circ} \mathrm{C}-165^{\circ} \mathrm{C}=5^{\circ} \mathrm{C}\right)$ e também na pressão de prensagem $(4-3=1 \mathrm{MPa})$ impactam em um reduções significativas de tempo, energia e até em mão de obra especializada na escala do processo desses materiais compósitos.

\section{CONCLUSÕES}

Com base nos resultados apresentados na presente pesquisa, pode-se concluir:

- A maioria dos tratamentos atendeu ao requisito normativo das propriedades físicas e mecânicas, com destaque ao tratamento aos tratamentos 10 e 12, que atenderam aos requisitos para todas as propriedades avaliadas;

- Os fatores de produção avaliados (densidade nominal, pressão e temperatura) e suas respectivas combinações não são, para algumas propriedades, significativos, o que implica no uso de fatores que utilizem menos recursos, como energia e equipamentos;

- Para as propriedades onde os fatores de produção foram significativos pela análise estatística, o aumento dos fatores pode levar a uma menor performance do painel, o que implica que um menor uso de recursos pode levar a um desempenho melhor do painel e atendimento dos requisitos normativos.

Considerando os dados apresentados nesta pesquisa, os fatores de produção avaliados podem levar, 
quando alterados, a uma melhor obtenção de propriedades que atendam aos requisitos normativos. Para resultados mais gerais, faz-se necessário estudos versando com partículas de outras espécies de madeira, bem como a adição de outras temperaturas e pressões como fatores de produção.

\section{AGRADECIMENTOS}

Os autores agradecem a Coordenação de Aperfeiçoamento de Pessoal de Nível Superior (CAPES) e ao Conselho Nacional de Desenvolvimento Científico e Tecnológico ( $\mathrm{CNPq}$ ) pelo apoio no desenvolvimento das pesquisas realizadas.

\section{BIBLIOGRAFIA}

[1] AQUINO, V.B.M., ALMEIDA, D.H., ALMEIDA, T.H., et al., "Physical and Mechanical Characterization of Cedrelinga catenaeformis Ducke Wood Specie", International Journal of Materials Engineering, v. 8, n. 5, pp. 97-100, 2018.

[2] DIAS, F.M., LAHR, F.A.R., "Estimativa de propriedades de resistência e rigidez da madeira através da densidade aparente", Scientia Forestalis, n. 65, pp.102-113, 2004.

[3] YAMANAKA, S., MAGARA, K., HIRABAYASHI,Y., et al., "Reduction of formaldehyde emission from plywood using composite resin composed of resorcinol-formaldehyde and urea-modified scallop shell nanoparticles", Wood Science and Technology, v. 51, n. 2, pp. 297-308, 2017. doi:10.1007/s00226-0160868-3.

[4] GORSKI, L., BAYESTORFF, A., RIOS, P.D.A., "Produção de painéis de partículas orientadas ( OSB ) de Eucalyptus benthamii e Pinus spp . em diferentes combinações de camadas Production of oriented strand board ( OSB ) from Eucalyptus benthamii and Pinus spp wood at different lay", Scientia Forestalis, v. 43, n. 106, pp. 367-375, 2015.

[5] SILVA, J.V.F., FERREIRA, B.S., CAMPOS, C.I., et al., "Characterization of particleboards produced with Pinus spp. waste”, Scientia Forestalis, v. 44, n. 111, pp. 623-628, 2016.

[6] THOMPSON, R.J.H., ANSELL, M.P., BONFIELD, P.W., et al., "Fatigue in wood-based panels. Part 1: The strength variability and fatigue performance of OSB, chipboard and MDF", Wood Science and Technology, v. 36, n. 3, pp. 255-269, 2002.

[7] AKGÜL, M., UNER, B., ÇAMLIBEL, O., et al., "Manufacture of medium density fiberboard (MDF) panels from agribased lignocellulosic biomass", Wood Research, v. 62, n. 4, pp. 615-624, 2017.

[8] MUTTIL, N., RAVICHADRA, G., BIGGER, S.W., et al., "Comparative Study of Bond Strength of Formaldehyde and Soya based Adhesive in Wood Fibre Plywood”, Procedia Materials Science, v. 6, pp. 2-9, 2014.

[9] CARVALHO, A.G., MORI, F.A., MENDES, R.F., et al., "Use of tannin adhesive from Stryphnodendron adstringens (Mart.) Coville in the production of OSB panels", European Journal of Wood and Wood Products,v. 72, n. 4, pp. 425-432, 2014.

[10] GARZÓN-BARREIRO, N.M., SHIRAKAWA, M.A., BRAZOLIN, S., et al., "Evaluation of mold growth on sugarcane bagasse particleboards in natural exposure and in accelerated test", International Biodeterioration and Biodegradation, v. 115, pp. 266-276, 2016.

[11] MANTANIS, G.I., AYHANASSIADOU, E.T., BARBU, M.C., et al., "Adhesive systems used in the European particleboard, MDF and OSB industries", Wood Material Science and Engineering, v. 13, n. 2, pp. 104-116, 2018.

[12] ZHOU, X., PIZZI, A., "Pine tannin based adhesive mixes for plywood", International Wood Products Journal, v. 5, n. 1, pp. 27-32, 2014.

[13] FERREIRA, B.S., SILVA, M.S., CAMPOS, C.I., "Análise do desempenho físico-mecânico de compensados produzidos com adesivos a base de PVA", Madeira Arquitetura e Engenharia, v. 12, pp. 13-22, 2011.

[14] SOUZA, A.M., VARANDA, L.D., MACEDO, L.B., et al., "Mechanical Properties of OSB Wood Composites with Resin Derived from a Renewable Natural Resource", International Journal of Composite Materials, v. 4, n. 3, pp. 157-161, 2014.

[15] BERTOLINI, M.S., CHRISTOFORO, A.L., CALIL NETO, C., et al., "Particulate composites with wastes from treated wood and tire rubber", Advanced Materials Research, v. 1025-1026, pp. 288-291, 2014. 
[16] PAN, Z., ZHENG, Y., ZHANG, R., et al., "Physical properties of thin particleboard made from saline eucalyptus", Industrial Crops Products, v. 26, n. 2, pp. 185-194, 2007.

[17] MACEDO, L.B., AQUINO, V.B.M., WOLENSKI, A.R.V., et al., "Paineis híbridos de lâminas e partículas de madeira para uso estrutural", Ambiente Construído, v. 19, n. 3, pp. 15-23, 2019.

[18] OLIVEIRA, S.L., FREIRE, T.P., MENDES, L.M., et al., "The Effect of Post-Heat Treatment in MDF Panels", Materials Research, v. 20, n. 1, pp. 183-190, 2017.

[19] GONÇALVES, C., PAIVA, N.T., FERRA, J.M., et al., "Utilization and characterization of amino resins for the production of wood-based panels with emphasis on particleboards (PB) and medium density fibreboards (MDF). A review", Holzforschung, v. 72, n. 8, pp. 653-671, 2018.

[20] IWAKIRI, S., TRIANOSKI, R., FONTE, A.P.N., et al., "Potencial de uso de madeiras de Dinizia excelsa Ducke e Protium puncticulatum J . F . Macbr para produção de painéis EGP”, Scientia Forestalis, v. 44, n. 111, pp. 709-717, 2016.

[21] IWAKIRI, S., CAPRARA, A.C., SAKS, D.C.O., et al., "Produção de painéis de madeira aglomerada de alta densificação com diferentes tipos de resinas", Scientia Forestalis, n. 68, pp. 39-43, 2005.

[22] BERTOLINI, M.S., NASCIMENTO, M.F., CHRISTOFORO, A.L., et al., "Paineis de Partículas Provenientes de Rejeitos de Pinus sp. Tratado com Preservante CCA e Resina Derivada de Biomassa", Revista Árvore, v. 38, n. 2, pp. 339-346, 2014.

[23] CHIROMITO, E.M.S., CAMPOS, C.I., FERREIRA, B.S., et al., "Propriedades mecânicas de painéis produzidos com lascas de madeira em três diferentes comprimentos", Scientia Forestalis, v. 44, n. 109, pp. $175-180,2016$.

[24] FERRO, F.S., ALMEIDA, T.H., SOUZA, A.M., et al., "Painel híbrido OSB/MDP de madeira Pinus taeda e resina poliuretana à base de óleo de mamona", Ambiente Construído. v. 19, n. 3, pp. 7-14, 2019.

[25] PALMA, H.A.L., BALLARIN, A.W., "Propriedades físicas e mecânicas de paineis LVL de Eucalyptus grandis", Ciencia Florestal, v. 21, n. 3, pp. 559-566, 2011.

[26] MOLINA, J.C., CALIL JUNIOR, C., "Sistema construtivo em "wood frame" para casas de madeira", Seminário de Ciências Exatas e Tecnológicas, v. 31, pp. 143, 2010.

[27] NOGUEIRA, M.C.J.A., ALMEIDA, D.H., ARAUJO, V.A., et al., "Physical and mechanical properties of Eucalyptus saligna wood for timber structures", Ambiente Construído, v. 19, n. 2, pp. 233-239, 2019.

[28] LOBÃO, M.S., DELLA LUCIA, R.M., MOREIRA, M.S.S., et al., "Caracterização das Propriedades Fisico-Mecânicas da Madeira de Eucalipto com Diferentes Densidades", Sociedade de Investigações Florestais, v. 28, n. 6, pp. 889-894, 2004.

[29] ZANUNCIO, A.J.V., MOTTA, J.P., SILVEIRA, T.A., et al., "Physical and Colorimetric Changes in Eucalyptus grandis Wood after Heat Treatment”, BioResources, v. 9, n. 1, pp. 293-302, 2014.

[30] SHIROSAKI, R.K., ALMEIDA, T.H., PANZERA, T.H., et al., "Caracterização de painéis de partículas de média densidade feitos com resina poliuretana monocomponente à base de mamona", Ambiente Construído, v. 19, n. 1, pp. 37-43, 2018.

[31] ALMEIDA, T.H., ALMEIDA, D.H., ARROYO, F.N., et al., "Time of exposure at $60{ }^{\circ} \mathrm{C}$ service temperature: Influence on strength and modulus of elasticity in compression parallel to the grain of hardwood species", BioResources, v. 14, n. 1, pp. 207-219, 2019.

[32] ABNT, Chapas de madeira aglomerada - NBR 14810, 2013.

[33] C. Standard, Mat formed wood particleboard - CS 236-66, 1968.

[34] E.C. for Standardization, Particleboard: specifications - EN 312, 2003.

[35] LONGO, B.L., CUNHA, A.B., RIOS, P.D., et al., "Caracterização tecnológica de painéis particulados produzidos com resíduos de cinco espécies tropicais", Scientia Forestalis, v. 43, n. 108, pp. 907-917, 2015.

[36] IHNÁT, V., LÜBKE, H., RUSS, A., et al., "Waste agglomerated wood materials as a secondary raw material for chipboards and fibreboards Part II. Preparation and characterization of wood chips in terms of their reuse", Wood Research, v. 62, n. 1, pp. 45-56, 2017.

[37] SCATOLINO, M.V., COSTA, A.O., GUIMARÃES JUNIOR, J.B., et al., "Eucalyptus wood and coffee parchment for particleboard production: Physical and mechanical properties", Ciência e Agrotecnologia, v. 41, n. 2, pp. 139-146, 2017. 
[38] SOUZA, A.M., NASCIMENTO, M.F., ALMEIDA, D.H., et al., "Wood-based composite made of wood waste and epoxy based ink-waste as adhesive: A cleaner production alternative", Journal of Cleaner Production, v. 193, pp. 549-562, 2018.

[39] SILVA, D.W., FARRAPO, C.L., RIBEIRO, D.P., et al., "MDP com partículas de eucalipto e palha de milho", Scientia Forestalis, v. 43, n. 108, pp. 853-862, 2015.

[40] NASCIMENTO, M.F., BERTOLINI, M.S., PANZERA, T.H., et al., "Painéis OSB fabricados com madeiras da caatinga do nordeste do Brasil", Ambiente Construído, v. 15, n. 1, pp. 41-48, 2015.

[41] CHAHARMAHALI, M., TAJVIDI, M., NAJAFI, S.K., "Mechanical properties of wood plastic composite panels made from waste fiberboard and particleboard", Polymer Composites, v. 29, n. 6, pp. 606610, 2008.

[42] ANDRADE, L.M.F., SCATOLINO, M.V., FARIA, D.L., et al., "Inclusão do resíduo de polpação da celulose na produção de painéis aglomerados de média densidade", Scientia Forestalis, v. 46, n. 120, pp. 626-637, 2019.

\section{ORCID}

Diogo Aparecido Lopes Silva Vinicius Borges de Moura Aquino Francisco Antonio Rocco Lahr André Luis Christoforo http://orcid.org/0000-0002-7514-7467

http://orcid.org/0000-0003-3483-7506

http://orcid.org/0000-0002-3510-8498

http://orcid.org/0000-0002-4066-080X 